\title{
BOUNDS FOR EXTREME ZEROS OF QUASI-ORTHOGONAL ULTRASPHERICAL POLYNOMIALS
}

\author{
KATHY DRIVER AND MARTIN E. Muldoon
}

Abstract. We discuss and compare upper and lower bounds obtained by two different methods for the positive zero of the ultraspherical polynomial $C_{n}^{(\lambda)}$ that is greater than 1 when $-3 / 2<$ $\lambda<-1 / 2$. Our first approach uses mixed three term recurrence relations and interlacing of zeros while the second approach uses a method going back to Euler and Rayleigh and already applied to Bessel functions and Laguerre and $q$-Laguerre polynomials. We use the bounds obtained by the second method to simplify the proof of the known interlacing of the zeros of $\left(1-x^{2}\right) C_{n}^{(\lambda)}$ and $C_{n+1}^{(\lambda)}$, for $-3 / 2<\lambda<-1 / 2$.

Mathematics subject classification (2010): 33C45. bounds.

Keywords and phrases: Quasi-orthogonal ultraspherical polynomials; Gegenbauer polynomials; zeros;

\section{REFERENCES}

[1] C. BREZINSKI, K. DRIVER AND M. Redivo-Zaglia, Quasi-orthogonality with applications to some families of classical orthogonal polynomials, Appl. Numer. Math. 48 (2004), 157-168.

[2] T.S. Chinara, On quasi-orthogonal polynomials, Proc. Amer. Math. Soc. 8 (1957), 765-767.

[3] D. Dickinson, On quasi-orthogonal polynomials, Proc. Amer. Math. Soc. 12 (1961), $185-194$.

[4] D. K. Dimitrov And G. K. Nikolov, Sharp bounds for the extreme zeros of classical orthogonal polynomials, J. Approx. Theory 162 no. 1 (2010), 1793-1804.

[5] A. DRAUX, On quasi-orthogonal polynomials, J. Approx. Theory 62 no.1 (1990), 1-14.

[6] K. DRIVER, Interlacing of zeros of Gegenbauer polynomials of non-consecutive degree from different sequences, Numer. Math. 120 (2012), 35-44.

[7] K. DRIVER AND P. DUREN, Trajectories of zeros of hypergeometric polynomials $F(-n, b ; 2 b ; z)$ for $b<-1 / 2$, Constr. Approx. 17 (2001), 169-179.

[8] K. DRIVER AND M. E. MuldoOn, Interlacing properties and bounds for zeros of some quasiorthogonal Laguerre polynomials, Comput. Methods Funct. Theory 15 (2015), 645-654.

[9] K. DRIVER AND M. E. Muldoon, Zeros of quasi-orthogonal ultraspherical polynomials, Indag. Math. 27 (2016), 930-944.

[10] L. FEJÉR, Mechanische Quadraturen mit positiven Cotesschen Zahlen, Math. Z. 37 (1933), $287-309$.

[11] D. P. GUPTA AND M. E. MuldoOn, Inequalities for the smallest zeros of Laguerre polynomials and their q-analogues, JIPAM. J. Inequal. Pure Appl. Math. 8 no. 1 (2007), Article 24.

[12] P. Hartman, Ordinary Differential Equations, Wiley, 1964.

[13] M. E. H. Ismail And M. E. Muldoon, Bounds for the small real and purely imaginary zeros of Bessel and related functions, Methods Appl. Anal. 2 (1995), 1-21.

[14] P. MARoni, Une caractérisation des polynômes orthogonaux semi-classiques. C. R. Acad. Sci. Paris, Sér. I 301 (1985), 269-272.

[15] P. Maroni, Prolégomènes à l'étude des polynômes orthogonaux semi-classiques, Ann. Mat. Pura Appl. IV Ser. 149 (1987), 165-183.

[16] P. MARoni, Une théorie algébrique des polynômes orthogonaux. Application aux polynômes orthogonaux semi-classiques, Orthogonal polynomials and their applications: Proceedings of the Third International Symposium (Erice, 1990), 95-130, IMACS Ann. Comput. Appl. Math, 9 (1991), Baltzer, Basel, 1991. 
[17] M. RieSZ, Sur le problème des moments, III, Ark. f. Mat., Astronom. och Fys. 17 no. 16 (1923), 1-52.

[18] J.A. Shонат, On mechanical quadratures, in particular, with positive coefficients, Trans. Amer. Math. Soc. 42 (1937), 461-496.

[19] G. SzEgő, Orthogonal Polynomials, American Mathematical Society Colloquium Publications, vol 23, 4th ed.,1975.

[20] G. N. Watson. A treatise on the Theory of Bessel Functions, 2nd ed., Cambridge University Press, 1944. 